\title{
An Uncommon Case of Filum Terminale Paraganglioma
}

\author{
Taraprasad Tripathy ${ }^{1}$, Ranjit Meher ${ }^{1}$, Aman Yusuf Khan ${ }^{1}$, Pranoy Paul ${ }^{2}$, Pranjal Phukan ${ }^{1}$, Arindom Kakati ${ }^{3}$
}

Departments of ${ }^{1}$ Radiology \& Imaging, ${ }^{2}$ Pathology and ${ }^{3}$ Neurosurgery, North Eastern Indira Gandhi Regional Institute of Health and Medical Sciences (NEIGRIHMS), Shillong-793018, India.

\author{
Corresponding Author: \\ Dr Taraprasad Tripathy \\ Email: taraprasad.mkcg@gmail.com \\ This is an Open Access article distributed \\ under the terms of the Creative Commons \\ Attribution License (creativecommons.org/ \\ licenses/by/3.0). \\ Received \\ Accepted \\ March 31, 2021 \\ Published \\ July 12,2021 \\ August 15, 2021
}

\begin{abstract}
Background: Paraganglioma is a rare benign neuroendocrine tumor of autonomic nervous system which is extremely uncommon at filum terminale. Case Report: We hereby discuss the clinico-patho-radiological features of a paraganglioma in a 29 -yearold male patient who presented with low backache and lower limb radicular symptoms. Conclusion: Although, there are no pathognomonic MR imaging findings, the presence of peripheral hypointense rim and prominent flow voids may give a clue to the diagnosis of paraganglioma.
\end{abstract}

Keywords: Cauda Equina, Low Back Pain, Magnetic Resonance Imaging, Paraganglioma, Radiography.

\section{Introduction}

Paragangliomas in the filum terminale are extremely rare benign, non-secretory neuroendocrine tumors [1]. Till date, only 33 cases have been reported in the international literatures [2]. A 29-year-old male patient presented to the Neurosurgical outpatient department with initial symptoms consisting of chronic low back pain for a period of nine months. He had also radicular pain in the right thigh. In this case report, an interesting case of filum terminale paraganglioma is being discussed in the light of clinico-patho-radiological features.

\section{Case Report}

A 29-year-old male presented with chief complains of chronic lower backache and radiating pain to the right thigh for 9 months. These were slow in onset and gradually progressing with time. The pain was aggravating on prolonged walking and not relieved by bending or standing position. Patient also had complained of hesitancy and incomplete urination with irregular bowel habits on and off. On clinical examination, the motor and sensory systems were within the normal limits. All the peripheral reflexes were present. The spine showed no deformity.

X-ray imaging of lumbosacral spine revealed no significant abnormalities. Following this, magnetic resonance imaging of the lumbosacral spine was done in the Department of Radiology and Imaging, NEIGRIHMS, Shillong before and after administration of gadolinium dimeglumine contrast. On T2- weighted sagittal scan, there was a well-defined round to oval shaped lesion measuring $1.5 \times 1.5 \times 1 \mathrm{~cm}$ noted at L2 vertebral body level. The lesion was extramedullary and intradural in location and appearing T2-hyperintense with hypointense peripheral ring [Fig.1a]. On T1-weighted images, the lesion appeared mildly hyperintense [Fig.1b]. There was no evidence of vertebral body scalloping or erosion. There was no neural foraminal extension or widening. On post-contract fat saturated T1weighted image, the lesion showed intense contrast enhancement with internal heterogeneity [Fig.1c,d]. As there were no signs and symptoms indicating catecholamine hypersecretion, such as hypertension, psychomotor distress or headache [1], 
laboratory tests to measure the levels of dopamine, epinephrine, noradrenaline and vanillymandelic acid were not done.

Under GA, the lesion was accessed by complete L2 with partial L1 and L3 laminectomy and complete excision of mass was performed. The lesion was well-encapsulated, globular, greyingwhite with elastic consistency and well vascularised adhering to the filum terminale. The caudal nerve root fibres were displaced by the lesion without any evidence of infiltration. No residual disease process was seen in post-operative MR imaging. The patient had an excellent post-operative recovery, and the symptoms regressed completely. The patient was discharged after a week.

On histopathological examination, gross appearance of the lesion was smooth and grey white on outer surface. Cut section showed solid, grey white with areas of hemorrhage. The lesion had thin capsule and showed nests of cells divided by fibrovascular septa. The tumor cells were round and polygonal arranged in vague Zellballen pattern, ribbons and cords invested by sustentacular cells [Fig.3a]. The chief (tumor) cells were uniform with occasional atypical cells. Nucleus had finely clumped chromatin and cell boundary was not well demarcated. There was mitosis of 6-8 per high power field. Special stain like alcian blue showed no positivity in tumor cells. Immunohistochemistry was positive for synaptophysin [Fig.3b] and chromogranin suggesting neuroendocrine tumor. The stains like GFAP (to rule outependymoma), pan-CK (to rule outpapillary adenocarcinoma) and vimentin (to rule outchondrosarcoma) were negative.

\section{Discussion}

Paraganglioma is a rare benign slowly growing neuroendocrine tumor which is derivative of embryonic sympathetic and parasympathetic nervous system [2]. It is of neuroectodermal in origin and was first described by Millerand

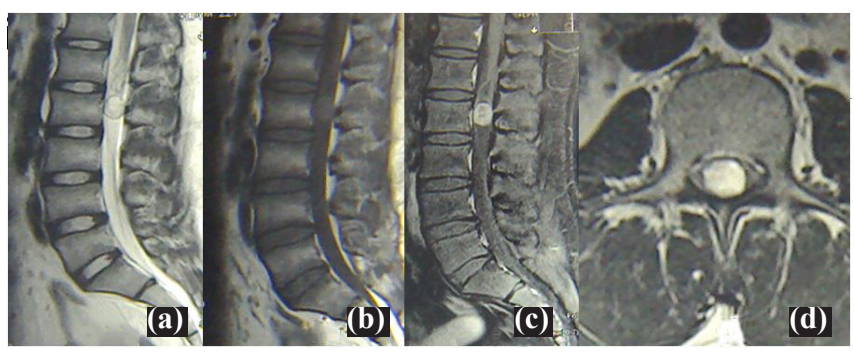

Fig.1:(a): Preoperative sagittal T2WI: Round to oval hyperintense lesion with hypointense periphery; (b): T1WI: Mildly hyperintense lesion; (c,d): T1FS + C: Intensely enhancing lesion with mild internal heterogeneity.

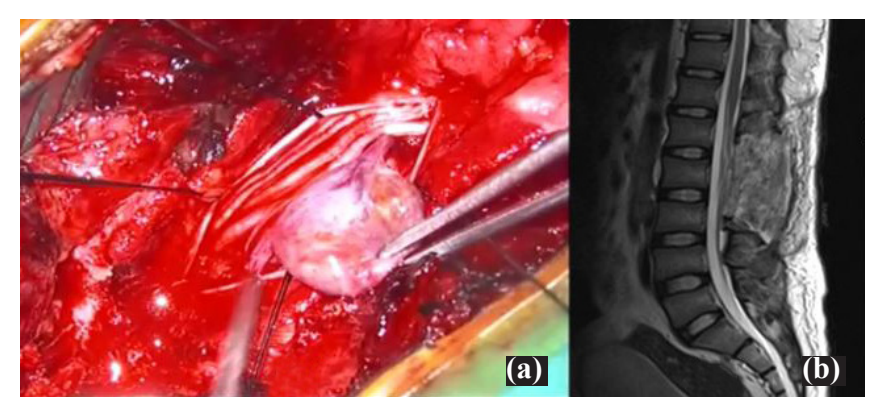

Fig.2:(a): Intraoperative findings: Well-encapsulated, globular, greying-white lesion with elastic consistency and well vascularised adhering to the filum terminale; (b): Postoperative MRI: No residual tumor tissue.

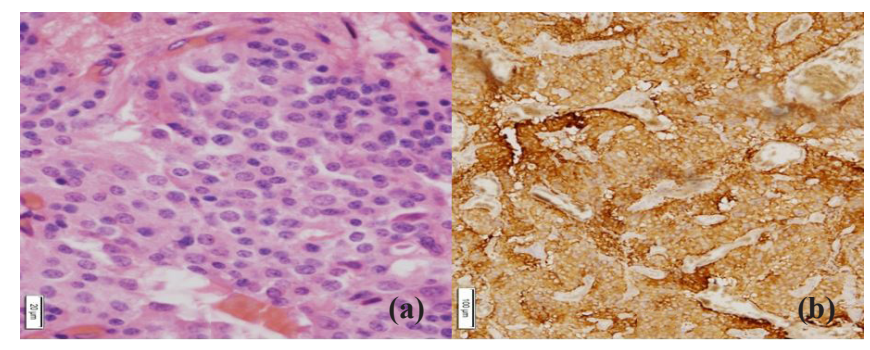

Fig.3:(a): Histopathology: Nests of cells consisting of round to polygonal cells arranged in vague Zellballen pattern, ribbons and cords invested by sustentacular cells; (b): Immunohistology: Synaptophysin positivity.

Torack in 1970 as secretory ependymoma [1]. The term paraganglioma was first named by Lerman in 1972 [3]. About $85-90 \%$ of the tumor arises from the adrenal gland and rest are extra-adrenal in origin. The most common extra-adrenal tumor is principally noted in the glomus jugulare of the carotid body [4]. The extra-adrenal tumors 
are usually multiple and non-secretory. Most of the lesions are sporadic. Few familial cases are associated with Von Hippel Lindau disease and MEN II. In the CNS, the most common location of paraganglioma is the petrous ridge, pineal gland and sella turcica $[5,6]$. The spinal paraganglioma is very uncommon, with an incidence of 0.07 per 100,000 general population and most commonly occur in the cauda equina and filum terminale region. There is a slight predominance in males. The tumor arises from sympathetic neurons in the lateral horn of the spinal cord and follows the course of the nerve roots $[7,8]$. Another possible locus of tumor origin consists of the heterotopic neurons that lie along the branches proximal to the sympathetic trunk. In the lumbar spine, the paraganglia situated in the cauda equina could be the origin of these lesions. However, it has been suggested [9] that ependymal cells play a role in the development of these lesions and do not derive from neural crest tissue. The cauda equina localization of paraganglioma has been well-documented. However, this tumor is much rarer in the filum terminale $[1,10,11]$ and few cases have been reported.

The tumor is usually seen in middle age with symptoms of low backache and radicular pain. This classical presentation was also seen in our patient. Cauda equina syndrome,sensory and motor deficit, and incontinence (urine and faeces) are unusual $[12,13]$. However, our patient had complained of hesitancy and incomplete urination with irregular bowel habits on and off. There are no pathognomonic MR imaging characteristics of paraganglioma, however it gives valuable anatomical information for the surgical planning and treatment. The lesion usually appears iso-intense on T1-weighted images and hyper-intense on T2-weighted sequences with prominent flow voids. There is intense enhancement after gadolinium contrast administration. These MR features are common to other intradural lesions, such as myxopapillary ependymoma, ependymoma, schwannoma and meningiomas [12,14]. The paraganglioma usually shows prominent flow voids which are uncommon in schwannoma and ependymoma. The T2-hypointense rim around the tumor is due to para-magnetic effects of hemosiderin resulting from repeated hemorrhage. In our case, the typical T2 hypointense rim was noted and no flow voids were seen. Currently, I-MIBG scintigraphy is preferred imaging method for localizing adrenal or extra-adrenal paragangliomas. Histopathological diagnosis of paraganglioma is based on the typical characteristics, namely an organoid or "Zellballen" patterns which consist of nests of argyrophilic cells with vascular stroma. Immunohistochemical testing shows a positive reaction for chromogranin and sustentacular cell immune reaction for S100 protein [10]. In our case there was strong positivity for synaptophysin and vimentin positivity in septa and capsule. In $10^{\text {th }}$ edition of International Classification of Disease for Oncology (ICD-O), cauda equina and filum terminale paragangliomas are classified as "tumours of neuro-epithelial tissue" in the sub-group of "Neuronal and Mixed Neuronal-Glial Tumours" (8680/1). In the WHO grading system, they are classified as grade $1[1,15]$. The prognosis of the tumor following complete resection is excellent. When complete removal is not feasible, radiotherapy maybe considered, however the role of chemoradiation has not been documented [10].

\section{Conclusion}

The spinal paraganglioma in the location of filum terminale is an extremely uncommon benign neuroendocrine tumor usually presenting with low back pain and radicular pain. There are no pathognomonic MR imaging findings however, peripheral hypointense rim and prominent flow voids provides a clue to the diagnosis of paraganglioma.

Contributors: TT, RM, AYK: manuscript writing, imaging; PPa: manuscript editing, histopathology; PPh: critical inputs into the manuscript; AK: manuscript editing, surgery. TT will act as a study guarantor. All authors approved the final version of this manuscript and are responsible for all aspects of this study.

Funding: None; Competing interests: None stated. 


\section{References}

1. Miller CA, Torack RM. Secretory ependymoma of the filum terminale. Acta Neuropathol (Berl). 1970;15:240250 .

2. Landi A, Tarantino R, Marotta N, Rocco P, Antonelli M, Salvati M, Delfini R. Paraganglioma of the filum terminale: case report; World Journal of Surgical Oncology. 2009; 7:95.

3. Bilbao JM, Horvath E, Kovacs K, Singer W, Hudson AR. Intrasellar paraganglioma associated with Hypopituitarism. Arch Pathol Lab Med. 1978;102:95-98.

4. Kruse F Jr. Petrous ridge chemodectoma (nonchromaffin paraganglioma) simulating meningioma. Case report J Neurosurg. 1960;17:1108-1111.

5. Smith WT, Hughes B, Ermocilla R. Chemodectoma of the pineal region, with observation on the pineal body and chemoreceptor tissue. J Pathol Bacterial. 1966;92:69-76.

6. Warrier S, Owler BK, Besser M. Paraganglioma and paragangliomatosis of the cauda equine. ANZ J Surg. 2006;76:1033-1037.

7. Seung-Yeob Y, Yong JJ, Sung HP, Tae AJ, Hyun JK, Chun KC. Paragangliomasin the cauda equine region: clinicopathoradiological findings in four cases. J Neurooncol. 2005;72:49-55.

8. Sonneland PR, Scheithauer BW, LeChago J, Crawford BG, Onofrio BM. Paraganglioma of the cauda equine region. Clinico-pathologic study of 31 cases with special reference to immunocytology and ultrastructure. Cancer. 1986;58:1720-1735.

9. Aghakhany N, George B, Parker F. Paraganglioma of the caudaequine region - report of two cases and review of the literature. Acta Neuroch. 1999;141:81-87.

10. Ashkenazy E, Onesti ST, Kader A, Llena JF. Paraganglioma of the filum terminale: Case report and literature review. J Spinal Disord. 1998;11(6):540-542.

11. Sousa J, O'Brien D, Crooks D. Paraganglioma of the filum terminale. J Clin Neurosci. 2005;12(5):584-585.

12. Boncoeur-Martel MP, Lesort A, Moreau JJ, Labrousse F, Roche I, Bouillet $\mathrm{P}$, et al. MRI of paraganglioma of the filum terminale. J Comput Assist Tomogr. 1996;20:162165.

13. Singh RV, Yeh JS, Broome JC. Paraganglioma of the caudaequine: a case report and review of literature. Clin Neurol Neurosurg.1993;95:109-113.

14. Levy RA. Paraganglioma of the filum terminale: MR findings. AJR Am J Roentgenol. 1993;160:851-852.

15. Louis D, Perry A, Reifenberger G, von Deimling A, Figarella-Branger D, Cavenee W, et al. The 2016 World Health Organization classification of tumors of the central nervous system: a summary. Acta Neuropathologica. 2016;131(6):803-820. 